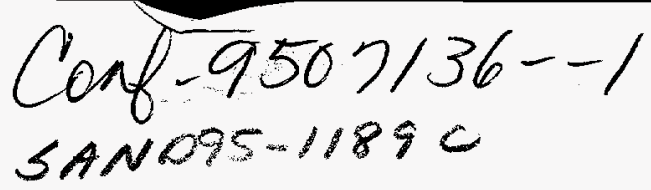

\title{
A Proposed Methodology for Computational Fluid Dynamics Code Verification, Calibration, and Validation
}

\author{
D.P. Aeschliman, W.L. Oberkampf, and F.G. Blottner \\ Aerosciences \& Fluid Dynamics Department \\ Sandia National Laboratories \\ Albuquerque, NM 87185
}

\begin{abstract}
Verification, calibration, and validation (VCV) of Computational Fluid Dynamics (CFD) codes is an essential element of the code development process. The exact manner in which code VCV activities are planned and conducted, however, is critically important. It is suggested that the way in which code validation, in particular, is often conducted-by comparison to published experimental data obtained for other purposes--is in general difficult and unsatisfactory, and that a different approach is required.

This paper describes a proposed methodology for CFD code VCV that meets the technical requirements and is phitosophically consistent with code development needs. The proposed methodology stresses teamwork and cooperation between code developers and experimentalists throughout the VCV process, and takes advantage of certain synergisms between CFD and experiment. A novel approach to uncertainty analysis is described which can both distinguish between and quantify various types of experimental error, and whose attributes are used to help define an appropriate experimental design for code VCV experiments. The methodology is demonstrated with an example of laminar, hypersonic, near perfect gas, 3-dimensional flow over a sliced sphere/cone of varying geometrical complexity.
\end{abstract}

\section{Introduction}

In the past, flight vehicle design and development have been based primarily on wind tunnel experimentation and flight testing. Mathematical methods, primarily approximate analytical solutions, have also made important contributions to design and development, but these methods were commonly directed toward improving the understanding of the flow physics or toward developing approximate engineering solutions. Modern Computational Fluid Dynamics (CFD) has evolved over roughly the past thirty years, tracking the availability of ever more capable computing hardware and algorithms. During much of that time, CFD has concentrated on the development of improved numerical algorithms and the solution of relatively simple research problems. More recently, a broader range of complex flow physics has been addressed along with advanced grid generation techniques for more complex and realistic geometries. As a result of the recent advent of massively parallel (MP) machines, highest available computing speeds now exceed 280 gigaflops (billions of floating point operations per second), and total random access memory now approaches 400 gigabytes, both numbers far in excess of projections for the mid-1990's made in 1983 by the National Research Council [1]. However, actual implementation of MP computing has been retarded by the additional effort required in writing code for MP architectures.

CFD has, in specific areas, begun to make important contributions to the design and development of aircraft, missiles, reentry vehicles, gas turbines, compressors, and rockets, to name a few. In addition, CFD codes are being used increasingly to describe complex fluid flow processes such as Chemical Vapor Deposition, shockboundary layer interactions, non-equilibrium reacting flows, freeshear-layer mixing, etc. However, the underlying physics of certain flow processes (e.g., boundary layer transition and turbulence) is still poorly understood. For such fluid mechanic processes, a predictive capability based on first principles is not available, and it is not entirely clear that simply increasing computing power will lead to valid solutions in those areas.

Just how valid are CFD codes and the solutions generated by them? -To some extent ignored by the CFD community in the past, the question of validity (accuracy and reliability) of CFD code predictions is now becoming critically important. CFD is being applied to the design of actual hardware, and a failure to answer the question of code validity is increasingly unacceptable.

Over the past decade, the critical and growing importance of this issue has been noted by numerous researchers.[2-7] In 1994 Oberkampf [6] presented a proposed framework for evaluating solutions from CFD codes, describing the particular types and classes of problems and the corresponding types of investigations needed to verify, calibrate, or validate codes designed to solve them. He concentrated on the broad philosophy of code verification ("Solving the governing equations right") and validation ("Solving the right governing equations"), definitions originally suggested by Boehm [8] and popularized by Blottner [9]. The terms "calibration" and "certification" also enter into this discussion. We loosely interpret code "calibration" to mean a code's ability to reproduce valid data (not exclusively experimental) over a specified range of parameters, for some geometry, without necessarily assessing the overall correctness of all of the physical models employed. We consider calibration to be a less-demanding element of validation, and is addressable experimentally by the same methods. "Certification" was defined by Mehta [10], as the entire process of establishing the credibility of a code, i. e., a certified code has been verified, calibrated, and validated.

Oberkampf [6] noted the importance and potential contributions of analytical solutions and other CFD solutions in addition to experiment as' sources of comparative input in the verification/ calibration/validation (VCV) process. This view is somewhat different from that proposed by NASA and summarized by Bradley [2] which considered comparison to experiment as the only acceptable method of CFD code validation. We, along with others (see, for example, [11]) take a somewhat softer stance regarding a total reliance on experiment. We are of the opinion that data from any source are appropriate for VCV purposes so long as they have been shown to be of high quality, and represent an appropriate test of the code, that is, they are based on the right physics or mathematical model. Thus, we believe that sources other than experiment can be used for validation purposes given the proper circumstances 


\section{DISCLAIMER}

This report was prepared as an account of work sponsored by an agency of the United States Government. Neither the United States Government nor any agency thereof, nor any of their employees, make any warranty, express or implied, or assumes any legal liability or responsibility for the accuracy, completeness, or usefulness of any information, apparatus, product, or process disclosed, or represents that its use would not infringe privately owned rights. Reference herein to any specific commercial product, process, or service by trade name, trademark, manufacturer, or otherwise does not necessarily constitute or imply its endorsement, recommendation, or favoring by the United States Government or any agency thereof. The views and opinions of authors expressed herein do not necessarily state or reflect those of the United States Government or any agency thereof. 


\section{DISCLAIMER}

Portions of this document may be illegible in electronic image products. Images are produced from the best available original document. 
For the verification part of this process ("solving the equations right") we are inclined to use comparison to exact analytic solutions or results from previously verified codes, and secondarily comparisons to experimental data. Conversely, for CFD code validation ("solving the right equations"), we believe primary reliance should be on comparison to carefully designed and conducted experiments, and less so on comparison to exact analytic solutions and results from other codes, a recommendation which, of course, applies to code calibration procedures as well. These distinctions are not sharply defined, and the proper approach should be determined on a case-by-case basis. At times there may be no choice. Certain processes cannot be adequately modeled experimentally, and the only recourse is through numerical simulation; examples include the flow inside a nuclear detonation fireball, planetary re-entry, and complex time-dependent multi-phase, multipath processes during a postulated nuclear reactor accident. If one accepts the definition of code validation given by Bradley[2], it follows that codes modeling such processes can be verified, and perhaps calibrated, but not validated. The issue of what constitutes verification, calibration, and validation of computer codes remains the subject of legitimate debate.

This paper describes our approach to the CFD code VCV process. We limit the scope to a brief outline of verification issues, and concentrate on code validation by experimental means. Our methodology consists of broad philosophical guidelines and specific procedural recommendations consistent with those guidelines by which the process is implemented. We will start by reviewing some of the historical circumstances which have influenced progress in this area to date, and discuss in some detail why we believe the CFD code VCV process must be an integral component of CFD code development, and not an "add-on" conducted after-the-fact. We will note certain inherent synergisms that, if identified and properly utilized, can lead not only to continued improvements in CFD code capability and credibility, but have the potential to improve experimental capabilities as well. We will describe a novel technique for uncertainty analysis and experimental design that serves to distinguish and quantify various sources of experimental error, and then present an example to demonstrate the methodology.

\section{Historical Background}

As noted above, Computational Fluid Dynamics has evolved more or less in parallel with the development of digital computers over approximately the past thirty years. Due to limitations in computer speed and memory, the early emphasis was on the development of numerical algorithms for simple physical models (inviscid flow over slender bodies of revolution at low angle of attack, for example), and was largely a research exercise. Computing speed has risen, on average, by a factor of ten every six years or so since 1960 , cost per compute cycle has fallen by a factor of at least $10^{3}$ and algorithm efficiency has improved by a similar factor. Over the same period computer memory has grown by a factor of $\approx 10^{5}$ for mainframes, and $\approx 10^{7}$ for the largest massively parallel machines (Paragon). This growth has enabled CFD to change from a research activity to an applied technology directed toward solutions to complex fluid engineering problems.

Throughout this period, code development has proceeded along a path largely independent of experimental validation. There are presumably diverse reasons for a lack of perceived need for code VCV and a concomitant lack of cooperation between the code development and experimental camps, but clearly an important contributor was the 1975 article by Chapman, Mark, and Pirtle.[12] That article predicted that CFD would be capable of solving all the important problems in fluid dynamics by the mid-1980s, and would eliminate the need for wind tunnel testing at that time. Chapman, et al, suggested that it might take a little longer if computing power lagged behind projections. (In fact, as noted earlier, growth in computing power has exceeded projections, but a first-principles computation of the "tough nuts" of fluid mechanics, transition and turbulence at high Reynolds number, for example, has yet to be demonstrated.)

Just at the time that key demonstrations of teamwork and cooperation might have shown the benefits of integrating VCV into the code development process, their paper contributed to polarizing computational and experimental fluid dynamicists into adversarial groups in sometimes bitter competition. In addition, U.S. policy makers in government appeared to take the views of Chapman, et. al., to heart (the effect appears to have been less profound outside the U.S.), resulting in an over-reliance on CFD code development, and a de-emphasis on new experimental capability development, especially new facilities.

There has, however, over the last five to seven years been a growing awareness $[2-4,6,7,13]$ on the part of experienced practitioners of both arts that such intense competition has not best served the interests of either group. To a large extent, this situation persists to this day, and effective implementation of a cooperative atmosphere, however desirable it may be, remains in general a significant challenge. Bad feelings and lack of trust are still as much the rule as the exception. Some organizations would still appear to have separate groups of CFDers and experimentalists who prefer not to talk to each other, and where cooperation does occur, it seems to be due to small teams forming voluntarily. It doesn't help that the majority of graduate students in fluid mechanics now concentrate on numerical simulation, and typically leave the university with little exposure to, and perhaps even less appreciation of physical experiments. To further compound the problem; many undergraduate schools in the U.S., presumably as a cost-cutting measure (we sincerely hope it is not because they feel it provides a better learning experience), now conduct their lab "experiments" on a PC. It is thus possible for students in physics or engineering to go through their entire academic careers and never witness, let alone actively participate in, an actual experiment. Modelers who hove no laboratory experience are unlikely to appreciate the true complexity of the real world.

\section{CFD at a Crossroads}

In what we believe was a landmark presentation, Dwoyer[13] in 1992 noted that CFD code development has come to a critical juncture, and in the absence of cooperation with, and key input from, those in other technical disciplines, is unlikely to make significant advances in attacking the really "tough nuts" of fluid mechanics. We emphatically agree. Specialists in other needed disciplines include computer scientists for new computer architectures; mathematicians for improved understanding of nonlinear analysis, for example, issues of well-posedness of the PDEs and boundary conditions; programmers for improved computational efficiency; molecular physicists and chemists to provide new data and improved descriptions of real gas effects, transport phenomena, and finite rate chemistry; theoretical fluid dynamicists to provide improved analytical insight, especially in the area of transition and turbulence modeling; experimental aerodynamicists and fluid physicists to obtain the appropriate flow and boundary condition data at the required accuracy for the code validation process; instrumentation specialists to develop advanced diagnostics concepts and measurement capabilities; facility designers and engineers for new or improved test facilities; and, of course, computational fluid dynamicists. Each of these disciplines must be intimately involved if CFD is ever to achieve its full potential. (One can argue that a successful computational fluid dynamicist will be, if not necessarily 
expert in each of the other disciplines, at least familiar with and have an appreciation of each of them.) Dwoyer referred to such an integrated activity as the "science of viscous aerodynamics."

Despite its obvious limitations the present capabilities of CFD are, of course, far from trivial. The advent of improved gridding techniques in finite element codes has greatly reduced the design cycle time for some problems. 2-D airfoils are designed by computer, not parametric wind tunnel test. Fast 3-D Euler solvers reliably predict steady high Reynolds number flow over simple wingbody-tail-pylon-engine configurations at low angle of attack. Further, we submit that for a somewhat narrowly restricted, welldefined set of problems, advanced CFD codes are now capable of producing results more accurate than can be obtained from, say, a wind tunnel experiment. Depending on the circumstances, the code may also be able to do it faster and more cheaply (although we find that meaningful cost comparisons are difficult to make, and are often misleading). An example which we have used in our own work, and which will be described more fully later in this paper, is laminar, perfect gas, hypersonic flow over a slender sphere/cone at low angle of attack. We now have sufficient confidence in certain CFD code predictions for this case that we use the results to provide an in-sin calibration of our hypersonic wind tunnel experiments. It is reasonable to expect that the range of problems that can be very accurately solved by CFD will continue to expand, especially given the barely-tapped potential of massively parallel computing.

Assuming it is true that CFD can compute certain flows better and faster than we can measure them, we see a changing relationship between CFD and wind tunnel experimentation. This changing relationship has been noted elsewhere $[14,15]$ in regard to wall and model support interference corrections for wind tunnel data. We believe that through teamwork and cooperation, this changing relationship can produce improvements in the capabilities of both computational and experimental fluid dynamics, and help to assure a future for both. Conversely, a continued 'them' vs. 'us' attitude will impede progress in both CFD and experiment, and will only serve to accelerate the already alarming pace at which aerospace test facilities are being abandoned.

The consequence of further decline in experimental capability is to us rather frightening, for it will necessarily imply an increasing dependence on new and unvalidated CFD codes for solutions to the most difficult remaining flow problems. The National Aerospace Plane, which was to be designed and developed with a very heavy reliance on $\mathrm{CFD}$, and the ensuing programmatic fiasco surrounding NASP after the truth became known, was not necessarily a unique event. We believe such a consequence is most certainly unwise, and is potentially catastrophic. We view it as axiomatic that CFD simply cannot do it alone, now or for the foreseeable future. Likewise, as we are all well aware, present experimental capabilities cannot provide a complete and simultaneous simulation of certain important flow regimes (e.g., high-enthalpy, high Reynolds number re-entry type flows), although as Mason [16], among others, has noted, very real progress in improving experimental capability continues to occur. Mason cites as examples improved connection of sub-scale testing to full-scale aerodynamics, advanced flow visualization, improvements in unsteady aerodynamics testing capability, and renewed emphasis on experimental foundations for advanced concept development. There is a proposal [17] by Prof. Miles and his colleagues at Princeton to examine in detail the technical feasibility of a new hypersonic facility that would provide a complete flow and chemistry simulation capability for Mach 10-20 flight at 100-200 thousand feet. Given the current funding climate, such a facility, even if feasible, is decades away. In the mean time, in the absence of the uitimate facility that "does it all", CFD is needed to help tie together experimental results obtained in a piece-meal fashion from separate facilities as they exist now, and to aid in advancing experimental capabilities in the future.

Some Computational Fluid Dynamicists go so far as to claim there is no need for verification, calibration, or validation of CFD codes. We would expect (and certainly hope) that such people represent a very small minority. A more common view among CFDers is that while code VCV is indeed necessary, the process-particularly the validation step--can be accomplished through comparison to existing data, documented in reports or archival journals, obtained for some other purpose than CFD code VCV. We strongly disagree. Almost invariably, critical details are missing from published data, particularly for archive journal data where discussion is typically limited in the interest of reducing paper length.

It is critically important that the boundary conditions assumed by the code be reproduced in the experiment. Rarely is such information presented in sufficient detail to ensure that BCs are matched. Wilcox ${ }^{18}$ quotes several examples that illustrate this point. In one case, turbulent heat transfer on an ablating nosetip with blowing was computed and compared to earlier published experimental data. Serious disagreement between the experimental data and the code predictions was seen. It later became possible to interact directly with the experimentalists and to discuss the experimental boundary conditions in detail. Once the proper experimental BCs had been input to the code, the agreement was excellent. In another case, initial agreement between experiment and code prediction for turbulent pipe flow was excellent. Later examinations of each showed that not only were the experimental results seriously in error, but that a substantial deficiency existed in the code physics. The original agreement was simply fortuitous. Such later opportunities are unusual, and may not suffice even if they can be arranged. Key personnel can become unavailable or forget important details, or there may be political or personal issues involved that make open and honest communication impossible.

\section{CFD Code Validity Issues}

The accuracy and reliability, i.e., validity, of CFD results depend on five elements: first, an accurate mathematical model of the important flow physics, including boundary conditions; second, accuracy of the discrete mathematical methods, e.g., difference methods, approximating the PDE's; third, adequacy of the mesh on which the discrete mathematical method, i.e., numerical algorithm, is solved; fourth, error free coding of the numerical algorithm; and fifth, adequate digital computing machinery. Overall CFD code utility additionally depends on ease of use and comprehension, e.g., visualization, of the computed results.

It is outside the scope of this paper to discuss code error sources in detail. However, the above five elements affecting code accuracy can be specified more precisely as:

a. Proper selection of the relevant flow physics and their mathematical representation

b. Accuracy of any auxiliary physical models (e.g., turbulence models)

c. Accuracy of the continuum boundary conditions

d. Discretization of the continuum equations (PDEs, physical models, BCs)

e. Spatial and temporal discretization convergence

f. Iterative convergence (per step or steady state)

g. Programming errors

h. Round-off/truncation errors

In general, code verification focuses on Items $d$ through $h$ ("solving the equations right"), i.e., on items related to numerical accuracy. Code calibration and validation deal primarily with Items 
a through $c_{2}$ ("solving the right equations"), and reflects the fidelity with which the code represents physical reality. Publication standards for articles in AIAA and ASME journals are now in effect regarding numerical accuracy of CFD code results $[19,20]$.

We close this discussion of CFD code validity with a comment about the Conservation Laws. Conservation of mass, momentum and energy are so ingrained that we tend to assume they are always true, whether a process occurs in nature or is simulated in a computer code. In a computer code, however, conservation must be demonstrated; it cannot be assumed just because mass, momentum and energy are conserved in the original partial differential equations. Loss of conservation can be caused by a wide variety of inaccuracies and/or errors in the numerical simulation, for example, inconsistency of the PDE's with the finite difference equations; lack of iterative convergence, either for a steady state solution or a time varying solution; the differencing schemes, and numerical limiters in those differencing schemes; artificial diffusion schemes; skewness of the structured or unstructured grid, etc. A related question is: does the numerical simulation conserve mass, momentum, and energy regardless of the grid size? This test is rarely applied in verifying computer codes.

\section{Philosophical Guidelines}

Our proposed CFD code VCV methodology is based on several broad philosophical guidelines. These guidelines have evolved from our own work and through our interactions with others. The underlying framework was presented in [6]. These guidelines are:

(1) A CFD code verification/validation experiment shall be jointly designed by experimentalists and CFD code developers working closely together thronghout the program, from inception to documentation, with complete candor as to the strengths and weaknesses of each approach. No withholding of limitations or deficiencies is permitted, and failure or success of any part of the effort must be felt by all. Without this level of cooperation and commitment, the process is very likely doomed to failure. Code VCV using published data obtained for other purposes is very risky and is strongly discouraged.

(2) $A C F D$ code verification/validation experiment shall be designed to capture the essential flow physics, including all relevant boundary conditions, assumed by the code. This is especially true for inflow/outflow $\mathrm{BCs}$, which directly impact whether, for example, a 2-D calculation is adequate, or a full 3-D solution is required. No physical experiment can be truly planar 2$\mathrm{D}$; there are only varying degrees of approximation of the actual 3-D flow so as to be approximately 2-D planar. There have been numerous examples where 2-D CFD simulations were computed to compare with a 'planar 2-D' experiment and it was found that the experiment had larger 3-D effects than expected. Generating planar 2-D flows for incompressible, low Reynolds number flow and for any Reynolds number in supersonic or hypersonic flow is very difficult.

Experimentalists must understand the code assumptions so that the experiment can match code requirements. Conversely, CFDers must understand the limitations of physical experiment, ensure that all the relevant physics are included, and work to define physically-realizable boundary conditions.

(3) A CFD code verification'validation experiment shall strive to emphasize inherent synergisms between the two approaches. For example, if sufficient confidence is available in a code solution for a simple flow physics case, computed results can be used as a calibration of the experiment. Other examples of such synergisms are noted later.
(4) Although the experimental design must be developed cooperatively, complete independence must be maintained in actually obtaining both the computational and experimental results. Neither side is permitted 'knobs' driving adjustable parameters. Only when the computed and experimental results are in hand is a comparative evaluation permitted, and only then is it appropriate to consider the causes of any differences.

(5) Invest in careful quantification of all relevant experimental parameters needed for comparison of predicted results to experiment. An obvious need, but routinely dismissed as too expensive, or too time consuming. Examples of essential data for a valid comparison of code results to experimental data are: freestream static conditions and flow angularity, inflow/outflow boundary conditions, wall and support interference effects, freestream turbulence intensity (for a transition or turbulent flow experiment), and body surface boundary conditions (surface temperature; measurements of actual departures from design shape, size, finish, waviness limitations, etc.) A decision is needed early-on as to what spatial resolution is necessary, particularly in regard to freestream property calibration. Although potentially high in initial cost, conducting this calibration step early may be cheaper, faster, and more reliable than going back later, when funds may be depleted, the facility or its staff may be unavailable, or experimental parameters may have changed in an unknown way.

(6) In general, conduct CFD code validation through a hierarchy of experiments of increasing difficulty and specificity. Start with easier experiments, then proceed to more complex and difficult ones, with each step providing an increasingly difficult challenge to the code. In wind tunnel experimentation, for example, a suggested hierarchy is:

a. Total body forces and moments

b. Control surface forces and moments

c. Surface pressure distributions

d. Surface heat flux and shear stress

e. Flow field distributions of pressure, temperature, and velocity components

f. Flow field distributions of Reynolds stresses

As the above hierarchy suggests, body forces and moments are the easiest of the physical quantities to both predict and to measure. Some have the view that total body forces and moments provide little or no value to the validation of CFD codes, arguing that because these data are such a gross measure of code accuracy, they provide little value to building confidence in a code's predictions. We contend that force and moment data are of value for two reasons. First, their value, i.e., their difficulty of prediction, depends directly on both the complexity of the flow physics and the complexity of the vehicle geometry. In cases of simple flow physics and simple geometries, we not only agree with the opposing viewpoint but we go beyond it. As stated earlier, these are cases for which we believe the flows can be computed more accurately than they can be measured. As a result, such flows can serve as experiment calibration cases for the experimentalist.

However, for somewhat more complex flow physics and vehicle geometries, the prediction of forces and moments can be more challenging than is commonly recognized. For example, Walker and Oberkampf [21] experienced substantial difficulty in predicting laminar flow body forces and moments on a reentry vehicle with a large flap deflection. Computing the large laminar separated flow region and reattachment on the flap proved at or beyond the present state of the art. Second, body forces and moments, as well as control surface forces and moments, can be measured more accurately than, say, surface heat flux. The experimental uncertainty bound on forces and moments is typically onetenth or less than that of surface heat flux. Therefore, the error 
tolerance on the $\mathrm{CFD}$ result nust also be a factor of ten better on the forces and moments as compared to heat flux to fall within the experimental uncertainty.

The general point is that as one progresses down the list to more difficult quantities for CFD to predict, the experimental uncertainty bound substantially increases also. In the process, knowledge is gained about the experiment that can lead to improved experimental technique and measurement accuracy in later, more difficult experiments.

(7) Employ an unceriainty analysis procedure that delineates and quantifies systematic and random error sources by type. Standard wind tunnel data uncertainty analysis typically attempts to quantify the statistical (random) uncertainty of individual components. It does not normally allow one to distinguish and quantify the contribution of one class of random error from another, nor to identify and quantify systematic errors. These might be random and/or systematic errors due to flow field nonuniformity or nonrepeatability, instrumentation uncertainties, and model geometry inaccuracies, for example. Once the error classes have been isolated, they can be analyzed using statistical techniques as recently described in [22], an extraordinarily detailed summary of uncertainty analysis as applied to wind tunnel testing. (Reference 22 also discusses typical sources of systematic errors and suggestions for reducing or eliminating them, but does not provide a method of identifying and quantifying them after the fact). Valuable information and insight regarding mathematical treatment of systematic (bias) errors is available in [23-25]. Our recommendations for specific steps in this process are presented in Sect 6 . Section 7 includes an example of data uncertainty analysis as applied to our own work.

\section{Experimental Methodology for CFD Code Validation}

How one goes about implementing the philosophical guidelines presented above is crucial to achieving satisfactory results. In implementing the proposed experimental methodology for CFD code validation we consider the following elements:

\section{Simulation Requirements}

A meaningful attempt to verify, calibrate, or validate a CFD code via experiment must begin with assessing the ability of the facility to simulate the flow and boundary conditions assumed by the code. If the parameters initially assumed for the calculation cannot be satisfied in the proposed experimental facility, it may be feasible to alter the code inputs so as to meet them, or it may be necessary to look elsewhere for a facility. For example, can the required boundary layer state on a model be assured? Is the type and quantity of instrumentation appropriate to provide the required data in sufficient quantity and at the required accuracy? We later use as an example the case of laminar flow over a three-dimensional hypersonic vehicle geometry, but the general approach should be similar regardless of the specific interest

\section{Synergisms between CFD and experiment}

By a 'synergism', we mean an activity whose primary intent is to meet a requirement for one approach, whether $\mathrm{CFD}$ or experiment, but which generates improvements in capability and/or accuracy of the other, such that both computational and experimental methods benefit. Particular synergisms will vary with the individual situation. Some examples of synergisms are:

a. If in a wind tunnel experiment one designs the wind tunnel model for easy modification from geometrically simple to complex, it becomes possible to produce a range of flow conditions from very simple, which can be calculated with high confidence, to very complicated flows which challenge or exceed the current computational state of the art. For example, for attached perfect gas laminar flow over a slender sphere/cone at low angle of attack, confidence in the computed solutions for flow over the simple model with simple flow physics can be such that the results are usable for an in-situ calibration of the freestream wind tunnel flow, thus increasing the efficiency and reducing the cost of the experimental investigation. For the flow over the more geometrically-complex region, the measurements can be used to validate the code (for example, shock/boundary layer interactions with laminar separation and reattachment). The reader may observe that such a calibration is not necessarily ideal, since it is sensitive to any variations in freestream properties over the volume occupied by the model. An alternative, but more expensive technique, is discussed below.) This synergistic use of the strength of one approach to offset a weakness of the other represents a powerful tool in the CFD code validation process.

b. A second synergism between CFD and experiment could be gained through an extension of the idea noted above. This would be to establish local inflow boundary conditions for the code via detailed local flow calibration measurements in a separate calibration experiment. This might be performed using minimally-intrusive mechanical or non-intrusive optical probes over a sufficiently fine spatial grid, and the resulting inflow data matrix used in computing the CFD solution over, say, a model in the test, volume. While such an approach might represent overkill at this stage of CFD code development for flows in typical (i.e., near perfect gas) wind tunnels of high flow quality, it would appear to be an essential requirement for code validation experiments in high enthalpy flow facilities in which non-uniform expansions combine with finite-rate chemistry. In such facilities, the local flow is typically very poorly characterized, making meaningful comparisons of experimental data to code predictions extremely difficult, if not impossible. Once such data had been acquired, however, the existence of a detailed flow calibration would be of substantial benefit to the facility for any other test or flow research purpose. (The alternative in this case would be to use the same or yet another complex CFD code to compute the inflow conditions, which somewhat defeats the purpose).

c. The coupled integration of CFD into operation of adaptive wall wind tunnels, and, especially, in correcting for wall and support interference on model aerodynamic data, is a synergism that has a large potential payoff if successful. It is desirable to test aircraft configurations at the largest possible scales to maximize Reynolds number, a goal which is in immediate conflict with minimizing interferences. The status of this activity has been recently assessed by Lynch, et. al., ${ }^{[14]}$ and Ashill [15] at the AGARD 73rd Fluid Dynamics Panel Symposium. Attempts to apply specific computational methodology are described by several authors at the same Symposium. It was noted by Lynch that the CFD capability required to compute interference corrections must advance in concert with the testing requirements.

d. A fourth potential synergism is the use of detailed, highprecision mechanical inspections to provide characterizations of model dimensions and surface roughness in order to specify the actual, as opposed to the design, wall boundary conditions for input to the code. Actual hardware cannot correspond exactly to design specifications, and these differences must be known for code validation purposes. (A qualification is that some imperfections in model dimensions may only be resolvable with very fine grids that could be too computationally expensive, and in the end may not improve the numerical prediction). Once the model imperfections are characterized, the model becomes a useful calibration tool for experimentation that may be unrelated to code validation. 


\section{Some Recommended CFD Code Validation Procedures}

The following specific procedures are recommended for implementing the methodology proposed in this paper for relatively long run-time code validation experiments. Use of short-duration (milliseconds or less) facilities such as shock tubes or shock tunnels would add a-strong temporal response and resolution requirement on experimentation, and unsteady flow CFD solution capability, including possibly unsteady boundary conditions in the code. The following list is by no means inclusive; different code validation experiments will necessarily generate different measurement issues.

a. Obtain detailed, accurate freestream flow calibration data at a spatial resolution consistent with code requirements. Freestream flow calibration at some level of sophistication is, of course, a requirement for even routine production wind tunnel testing. However, for CFD code validation purposes, flow field calibration must be done at typically finer spatial resolution, it must include all quantities required by the code as input boundary conditions, and the experimental uncertainties must be quantified. This is a daunting set of requirements, and can be very expensive and time consuming to meet. Further, for a turbulence or transition experiment, it must include a determination of freestream turbulence intensity and scale. It is axiomatic that most experimental facilities are not adequately calibrated in this context, either because the specific quantities were not needed for normal operations, or because of the high cost of acquiring measurements at the desired spatial density. Further, some facility managers may be reluctant to share such detailed flow quality data with users. However, for a CFD validation experiment it must be available. This is another argument for having, and using, one's own facilities for code validation research. Having total control over the facility is an invaluable advantage, and in some cases will be absolutely essential.

b. Precisely characterize the model wall boundary conditions, as tested. Differences will exist between the nominal and actual model dimensions, location, orientation, and surface condition. These must be known to an acceptable accuracy to provide wall boundary conditions for the code. Pre-test mechanical inspections of the model as assembled in all its possible configurations must include size, shape (e.g., straightness, out-of-round), surface finish (especially any steps at joints) and waviness. Aeroelastic effects must also be considered, since model and sting deflection under load can introduce systematic experimental errors well in excess of measurement precision, especially for aerodynamic forces and moments, and surface pressures.

If surface temperature can vary significantly, as in a longduration hypersonic flow experiment at non-zero angle of attack, and computed results are sensitive to surface temperature, then the model surface temperature distribution must be measured. If those temperature changes are both significant and non-uniform (3-D model, or any model at angle of attack), then shape change due to thermal expansion must be considered. (We have attempted to minimize this latter error through the use of low-expansion alloys, such as Invar-36, as a model material). Model orientation and location settings (angle of attack, roll angle, axial station) and configuration dimensions must be precisely determined, including the repeatability of these values if the model configuration will be altered routinely. These data will be important input for experimental error assessment.

c. Vary experiment scale in same facility at same nominal test conditions. This is a valuable strategy to ascertain wall or support interference effects, unsuspected Reynolds number effects such as incipient transition on the model, or limited core flow uniformity or extent. The penalties are added test and model costs, and depending on individual circumstances, not all model dimensions and tolerances may be directly scalable.

d. Conduct same experiment in different facilities. If feasible, conduct the same code validation experiment in more than one facility. Satisfactory agreement of results lends confidence that there are no inadequately understood facility-related bias errors in the data, e.g., condensation effects, excessive flow angularity, etc. The corollary to this, of course, is to use the results of different codes to predict the simple flow cases used for any in situ calibrations conducted in the experiment

e. Apply redundant measurement techniques for critical experimental variables. Since no measurement is free of error, and no single measurement technique is best for all applications and ranges of parameters, redundant measurements of critical variables should be performed whenever possible, and certainly if there is a suspicion that a measurement technique is of questionable applicability under some conditions. For example, suppose that one desires to use a pitot-static probe to calibrate the freestream Mach number over the test section. Suppose further that the Mach number and probe Reynolds number ranges for some experiments are such that viscous effects on probe accuracy may be important. A redundant measurement of freestream Mach number could be obtained by measuring the flow velocity and static temperature independently using nonintrusive techniques, and computing the Mach number.

f. Construct and apply an uncertainty analysis capable of delineating and quantifying all important error sources, e.g., flow field nomuniformities separated from errors due to instrumentation uncertainties and model inaccuracies. This is a key element of our methodology, and is distinctly different from, and goes significantly beyond, standard uncertainty analysis. Our method does use standard statistical methods but in addition incorporates several novel extensions of the standard methods. This is particularly true in regard to design of the experiment run matrix and the use of repeat runs, reflection of data around axes of model symmetry, and in situ freestream calibrations based on comparison to code predictions for cases of particularly simple model geometry and flow physics. In this way, random errors can be isolated from certain systematic errors in the course of the data uncertainty analysis, and both types of error can be quantified. An example application of this technique from our own work is given in Section 7 .

Detailed methodology for statistical error analysis as it applies to experimental data in general has been widely available for many years. ${ }^{26}$ A recently published treatise, by far the most detailed approach to dealing with systematic and random errors in wind tunnel data when the systematic errors have been previously identified and estimated, is presented in [22]. That document identifies virtually every conceivable source of experimental error in wind tunnel testing and greatly improves the art of wind tunnel data uncertainty analysis.

g. Design the experimental run matrix to support the uncertainty analysis. The run matrix should be carefully designed so that combinations of runs yield both statistical and bias error information. Using the technique of [27], the effects of individual transducers, model pitch angle, and roll orientation can be isolated from effects of flow field nonuniformity and model imperfections. The common technique of plotting data taken at positive angle of attack with the model at zero roll angle against data at negative angle of attack with the model rolled 180 degrees is an example of the approach. Any vertical flow angularity will be apparent from a combined plot of the data. This can be extended to all combinations of controlled experimental parameters. Statistical data is obtained through the use of selected repeat runs in which the controlled parameters are held constant (to within precision limits on repeatability, which of course contributes to the overall statistical uncertainty.) 
h. Specifically repeat runs in varying order, on different doys, and in different facility enlries. This strategy can uncover subtle errors related to facility operations, specific personnel, time of day, etc. Suppose, for example, that a particular sensor output is actually more temperature sensitive than expected, and that the ambient temperature routinely rises during the course of the day. Repeating runs in reverse order and comelating the results will detect errors resulting from this effect, in addition to providing random error estimates. Repeat runs in the proposed methodology are not afterthoughts. They require careful introspection in their selection and sequence, and are critical to a valid assessment of the absolute accuracy and statistical precision of the data. Repeat runs must be incorporated into the experimental plan and the results included in the experimental data set.

i. If a model has appropriaie geometric symmetry, plot all pitch data at positive angle of attack. This technique was alluded to above and takes advantage of certain symmetries to differentiate error sources from each other. Data obtained with a model at zero roll angle and pitched from, say, 0 to $+10 \mathrm{deg}$ angle of attack $\alpha$ can be plotted for positive $\alpha$ with data for a model at $180 \mathrm{deg}$ roll angle and pitched from $\alpha=0$ to $-10 \mathrm{deg}$. The result is that errors associated with model geometry can be separated from errors due to flow field nonuniformity, flow angularity in the vertical plane, for example. Following similar logic, flow angularity in the yaw plane can be quantified by appropriately reflecting data taken at 90 and $270 \mathrm{deg}$ roll angles.

j. Take and keep notes that are as careful, detailed, and extensive as possible. This is a truism, and its desirability is obvious to any experimentalist. From our own experience, however, it is far too easy to fail to record information that later proves to be important when trying to explain any anomalies that arise during the data analysis phase. This is, of course, especially true in regard to obviously unusual circumstances or events, but it applies to seemingly mundane or routine items as well. Insofar as understanding the experimental data is concerned, it is essentially impossible to record too mich annotative information.

Because it is our experience base, we have chosen to present the methodology in terms of wind tunnel experimentation in relatively long-duration (seconds or longer) aerospace testing facilities. However, extension of the more general recommendations to other experiments should be apparent. Implementation of these recommended procedures is, of course, not free. And some may not be practical in each situation, either technically or economically. With each step or procedure followed, however, the overall experimental uncertainty can be reduced and the quality of the code validation process improved.

Clearly, some of these recommendations are easier to implement than are others. The first recommendation--to acquire a complete, detailed, finely-spaced calibration of the tunnel freestream--represents an expensive, time-consuming exercise. For heavily utilized production facilities, interference with higher priority work may make such flow field calibrations extremely difficult to obtain. Even for research-oriented facilities for which interference with other work may not be a problem, performing such calibrations almost certainily will require a substantial investment. It is unfortunate that the recommendation that, in general, is probably the most important in conducting code validation experiments is the most difficult to fulfill.

But the situation is not necessarily all that bleak. In-situ calibrations based on CFD performance predictions for a simple geometry may provide a technically-acceptable alternative at minimal cost in typical aerospace simulation facilities for some, if not most, code validation experiments in facilities with high flow quality. That is, this approach will be satisfactory if the scale of the model is small relative to the variations in freestream properties over the model volume at the model location, which is the case for the work described here. Failing that, a possible conclusion may be that some facilities will be dedicated to production testing exclusively, for which existing calibrations and data bases are presumably already adequate, and others will be used to provide the needed code validation capability.

\section{A Case Study for CFD Code Validation Methodology}

\section{Experimental Design}

Since 1990, Sandia has been involved in a program, referred to as the Joint Computational/ Experimental Aerodynamics Program (JCEAP). The purpose of JCEAP is to improve both Sandia's wind tunnel experimentation and CFD development capabilities. This program will be described briefly in order to illustrate our code validation methodology. Representative experimental and computational results for body forces and moments, and surface pressure distributions will be presented for a model in laminar Mach 8 flow. A more detailed description of the force and moment measurements and their comparison to results computed using 3-D viscous flow codes are presented in [27].

For the code validation experimentation in JCEAP, we have used a 4-in. base diameter, 10-deg half-angle, 10-percent blunt, sting-mounted sphere/cone model with an aft slice parallel to the, axis, Fig. 1. The model length, $L$, is 10.39-in and the slice commences at the $70 \mathrm{~L}$ point. At the rear of the slice can be attached fixed flaps of variable deflection angle, 10,20 , and $30 \mathrm{deg}$. The trailing edge of the flaps extended to the model baseplane for all flap angles.

We have constructed two test models of nominally-identical external size and shape. One is the force and moment model described in [27], and is used in conjunction with a precision sixcomponent internal strain gage balance. The other is a pressure model equipped with two 48-port Electronically-Scanned Pressure modules mounted internal to the model in order to minimize pneumatic tubing lengths and pressure lag time. The pressure model is also provided with nine semiconductor-bridge Kulite gages to detect high-frequency pressure fluctuations should they occur. Also, six coaxial thermocouples were mounted in the model wall to provide the wall thermal boundary condition to the code. The pressure model and instrumentation, and some early test results are described in [28]. All model dimensions and pressure port locations were determined to $+/-0.0002$ inch via a detailed mechanical inspection.

For JCEAP we chose a body geometry that eliminates several potentially-troublesome numerical difficulties. For example, by requiring the body flap to extend to the base plane of the model for all flap deflections, a substantial simplification became possible in (a) defining the grids for the body geometry and for the base flow, and (b) in setting the outflow boundary conditions across the baseplane in the numerical simulation. Having the flap trailing edge extend to the baseplane for an arbitrary deflection angle would, of course, be unrealistic for hinged flaps on actual flight hardware.

It is relatively straightforward to experimentally measure model surface temperature. It is difficult, however, to fabricate complex models with uniform thermal properties. To compute the model surface temperature distribution as a function of time for a non-uniform model wall thickness would have placed a large additional computational requirement, largely unrelated to the fluid mechanics, on the code. Hence, the model was instrumented to provide this boundary condition for the computations.

We also chose to require that the flow on the model vehicle be laminar everywhere in order to avoid the uncertainty (and 
"adjustable knob") that would be introduced by use of a turbulence model. (In addition, this choice avoided the requirement for a detailed calibration of the freestream turbulence intensity level over the tunnel test section, an additional synergism of sorts). Flow visualization using shear-stress-sensitive liquid crystals $[27,29]$ was employed in a preliminary series of experiments with varying freestream Reynolds number in order to ensure that the boundary layer was, in fact, laminar over the entire model for all validation experiments. The liquid crystal technique also provided a wealth of surface flow characterization data for cases with massively separated flow on the flap.

Base pressure was carefully measured because of its relatively large contribution to axial force. A base-plate was attached to the sting (not the model, so as to avoid bridging the balance with pneumatic lines). Measurements of the pneumatic lag time of the base pressure instrumentation were used to set data acquisition delay time following a change in angle of attack using a pitch/pause sequence.

Nominal tunnel conditions for all experiments were as follows: stagnation pressure $P_{0}=340$ psia, stagnation temperature $\mathrm{T}_{\mathrm{O}}=1106 \mathrm{R}$, and freestream Reynolds number $\mathrm{Re}_{\infty}=2.0$ million/ft $\left(\operatorname{Re}_{\mathrm{L}}=1.80\right.$ million, based on model length). Angle of attack was varied from -10 to $+18 \mathrm{deg}$. Roll angle was set at 0 (slice on the windward side), 90,180 , or $270 \mathrm{deg}$. The primary purpose for the four roll angles was to quantify the effect on aerodynamic forces, moments, and surface pressures of flow field nonuniformities in the wind tunnel and the effect of model dimensional inaccuracies. The combination of multiple roll angles and angles of attack, and repeat runs at the same orientation and axial location in the tunnel were used to separate errors due to flow field nonuniformities and tunnel run-to-run repeatability from errors due to instrumentation uncertainties and model tolerances. Mirror symmetry was used to reflect data around axes of symmetry for varying pitch and roll angles. We also varied model axial location to assess possible errors due to flow axial gradients resulting from possible wave focusing in the axisymmetric nozzle.

\section{Uncertainty Analysis}

The uncertainty analysis is a critically-important element in our validation methodology. Our overall approach differs from that typically employed, not in the mathematical formulation but in that it is constructed around the use of various combinations of individual experiments to identify and separate errors due to different sources. Specifically, we are able through this technique to separate and quantify errors due to flow field nonuniformities, instrumentation, and model fabrication tolerances. From this statistical analysis emerges both random and bias error estimates for certain parameters (e.g., flow angularity). The absolute accuracy in the important freestream flow properties, such as Mach number and static pressure cannot be discerned from this analysis. As discussed previously, these data are determined via the in situ calibration against CFD predictions for the simple geometry and flow physics case. To limit the discussion, we will present the methodology for force and moment analysis; surface pressure error analysis, which also yields uncertainties due to model inaccuracies, is similar in principle, but differs in detail.

The instrumentation uncertainty is that uncertainty in body forces and moments, caused by all of the following: strain gage hysteresis, nonlinearity, thermal sensitivity shift, and thermal zero shift; the analog data acquisition system (amplifiers, A/D converters); the data recording system, including any digital filters; model pitch, roll, and yaw alignment; run-to-run variations in setting freestream conditions; and base pressure transducers and instrumentation for correcting for base axial force. To calculate the instrumentation uncertainty one compares body force and moment measurements for the model at the same physical location and orientation. From the run schedule one can choose run pairs that have the same pitch and roll angles and the same location, and then make comparisons between the measured body force and moment coefficients, and center of pressure. For a given run pair and for a particular coefficient, say axial force, the axial force coefficient $\overline{\mathrm{C}}_{\mathrm{a}}$ can be defined,

$$
\left(\overline{C_{a}}\right)_{\alpha_{i}}=1 / 2\left\{\left[\left(C_{a}\right)_{p}+\left(C_{a}\right)_{q}\right]_{\alpha_{i}}\right\}, i=1,2, \ldots \ldots . . I
$$

$p$ and $q$ represent the nun numbers from which the measurements are taken, $\alpha_{i}$ refers to the nominal angle of attack at which each force and moment measurement was made, and $I$ is the number of $\alpha$ 's, typically 12, which are in common for both run pairs. (Other force and moment coefficients and pressure coefficient are defined similarly.) Nominal steps in $\alpha$ were typically 3 degrees. Although known to $+/-0.01 \mathrm{deg}$, the exact values of $\alpha$ did not repeat from run to run due to jitter, typically $+/-0.1 \mathrm{deg}$. in the angle of attack drive mechanism; to compensate for this, the force and moment data were curve-fitted (7th-order spline) and the values adjusted slightly to common values of $\alpha$, which were chosen to be $0,-10,-7,-4,-1,2,5$, $8,11,14,17$, and 0 degrees.

Let the difference between an individual force and moment measurement and the average measurement at each angle of attack be defined as the local residual, $\Delta$,

$$
\left(\Delta \mathrm{C}_{\mathrm{a}}\right)_{\alpha_{i}}=\left(\mathrm{C}_{\mathrm{a}}\right)_{\alpha_{\mathrm{i}}}-\left(\overline{\mathrm{C}_{\mathrm{a}}}\right)_{\alpha_{\mathrm{i}}}, \mathrm{i}=1,2, \ldots . \mathrm{I}
$$

The uncertainty in body forces and moments due to a combination of instrumentation uncertainty and flow field nonuniformity can also be computed by comparing certain runs when the model is at physically different locations in the tunnel. There are two ways to do this. The first method forms run pairs that have the same roll and flap angles, but are at different axial stations, and is sensitive to axial flow gradient nonuniformity. The second method forms run pairs based on mirror symmetry between the model at roll angle $0 \mathrm{f} 0 \mathrm{deg}$ at a positive angle of attack, and with the model rolled $180 \mathrm{deg}$ and pitched to a negative angle of attack. Since the maximum negative $\alpha$ was $-10 \mathrm{deg}$, the residuals can be calculated only over the range -10 to $+10 \mathrm{deg}$ by this method.

Figure 2 shows the magnitude of the residuals $\Delta C_{a}$ in axial force coefficient versus the magnitude of $\mathrm{C}_{\mathrm{a}}$. This plot includes all of the residuals for both instrumentation and flow field nonuniformity components. The "quantized" character of the residuals is due to the truncation in the output data format of the evaluation of the curve fits to the data. "Axial Flow Gradient" in Fig. 2 refers to residuals calculated from flow field nonuniformity run pairs at different axial stations, and "Mirror Symmetry Flow" refers to run pairs formed from angle of attack symmetry at each axial station. It can be seen from Fig. 2 that the residuals for $C_{a}$, although very small in magnitude (roughly one percent of $C_{a}$ ) are dominated by flow field nonuniformity, not instrumentation uncertainty. The contributions from axial flow gradient and crossflow gradient contribute approximately equally.

The sample variance for the instrumentation component and also for the total experiment (combined instrumentation and flow field nonuniformity components) is calculated from

$$
\sigma^{2}=1 /(2 n) \sum_{j=1}^{n}\left[\Delta_{1}^{2}+\Delta_{2}^{2}\right]_{j}
$$

where $\mathrm{n}$ is the number of local residuals and $\Delta_{1}$ and $\Delta_{2}$ are the residuals for each run pair. These sample variances are estimates of corresponding instrumentation and combined "population variances." The relationship among the population variances is 


$$
\sigma_{\text {comb }}^{2}=\sigma^{2} \text { instrum }+\sigma^{2} \text { flow }
$$

because of the independence of instrumentation errors and flow field nonuniformities. Thus, given the sample variance due to instrumentation and that for the total experiment, the component due to flow field nonuniformity can be estimated by

$$
\sigma_{\text {flow }}^{2}=\sigma_{\text {comb }}^{2}-\sigma_{\text {instrum }}^{2}
$$

Table 1 gives the estimated standard deviation, $\sigma$, due to instrumentation, flow field nonuniformity, and the total for each coefficient (from left to right, normal force, pitching moment, center of pressure, and axial force) measured in the experiment. From Table 1 it can be seen that the uncertainty in normal force, pitching moment, and center of pressure due to the entire wind tunnel system instrumentation ranges from 9 to $20 \%$, whereas that due to flow field nonuniformity is 80 to $91 \%$. We imagine that these data are typical of facilities elsewhere, i.e., that most transonic through hypersonic wind tunnel experiments are dominated by flow field uncertainty and not instrumentation errors. The table also shows that uncertainty in forebody axial force coefficient is $63 \%$ due to instrumentation and $37 \%$ due to flow. This reversal of uncertainty contributions compared to the other quantities is most likely due to instrumentation inaccuracies in removing the base pressure component from the total axial force.

\section{Results}

Representative data for axial force coefficient, $C_{a}$, as a function of angle of attack are shown in Fig. 3 for the slice-only configuration. Agreement with parabolized Navier-Stokes (SPRINT) calculations [21] is excellent, 1-percent or better over the entire range of angle of attack. Since the CFD solutions for forces and moments are believed to have an absolute accuracy of 1-percent or better for the case of simple model geometry and flow physics at the defined freestream conditions, we conclude that the wind tunnel measurements of forces and moments are therefore calibrated to an equivalent level of absolute accuracy. This calibration is of great practical value, since it confirms the overall accuracy of the freestream properties (Mach number, Reynolds number, static pressure) assumed for the flow, based on the measured $P_{O}$ and $T_{O}$, and real-gas thermodynamic and transport property calculations for an isentropic expansion and nozzle boundary layer corrections.

Good $[\mathrm{O}(5-10 \%)]$ agreement between experiment and computation was observed for a flap angle, $\delta$, of $10^{\circ}$. For $\delta=20^{\circ}$ and $30^{\circ}$, however, large $[O(30 \%)]$ differences occurred between measurements and predictions. Figure 4 shows the measured axial force coefficient compared to the predictions using $F 3 D$ [30], a 3-D, time-iterative, first-order-accurate Navier-Stokes code, for the 30deg flap configuration. It was suggested previously [27] that the large discrepancies might be due to differences between the experimental and code outflow boundary conditions at the base of the model. Preliminary code calculations using INCA [31,32], a second-order-accurate, time-iterative, 3-D Navier-Stokes solver, indicate significantly better agreement $[\mathrm{O}(5 \%)]$ with measured force and moment data at the high flap angles for the same experimental conditions. This suggests that the improved agreement is largely a result of the increased numerical accuracy, and is not due to improperly assumed code boundary conditions.

The pressure distribution is a greater computational challenge than computing integrated forces and moments. As noted above, for the forebody, confidence in the computed results is high for the sphere/cone at low angle of attack. Calculating the expanding flow over the slice is expected to be somewhat more difficult, more so for some of the available codes than for others. Results for the surface pressure (absolute pressure measurement accuracy is typically $+/-0.001$ psia or better [28]) as a function of axial distance along the slice-only model for the windward (slice) and the leeward (cone) sides are shown in Fig. 5. Agreement between measured pressures and results computed using the inviscid 3-D flow code $S A N D I A C$ [33] is excellent on the cone, approximately $1 \%$, providing further confirmation of the overall accuracy of the in-situ calibration of the freestream flow. On the slice itself, however, measurable differences between the surface pressure measurements and predictions are already apparent for even this relatively simple case. The reduced pressure forward of the start of the slice relative to prediction is a result of the low pressure over the slice feeding forward in the subsonic portion of the boundary layer.

\section{Conclusion}

A proposed methodology for CFD code verification/ calibration/validation has been developed. The method incorporates specific experimental procedures that are consistent with, and an outgrowth of, a number of broad philosophical guidelines. Two guidelines are key: (a) a cooperative team effort between computational and experimental fluid dynamicists throughout the VCV program, from inception to documentation, and (b) use of a method of uncertainty analysis which assists in the experimental design, and which subsequently permits the delineation and quantification of various classes of both bias and random errors. The methodology has been demonstrated for hypersonic, near perfect gas flow over a sliced sphere/cone of variable geometry.

We reiterate our conviction that careful experiments designed specifically for code VCV purposes are an important, and usually the preferred, source of data for the calibration and validation portions of the VCV process. We expect that this will continue to be true for the foreseeable future. How future experiments are planned and conducted, however, is a significant issue. We hope the present work will provide useful guidance in this important area.

\section{Acknowledgments}

The authors thank the following Sandia National Laboratories personnel: J.F. Henfling, Z. Mahmud, R.E. Tate (ret.), D.R. MacKenzie (ret.) and C.B. Lafferty for their contributions to the experimental work; E.L. Clark for many helpful discussions; and D.E. Larson for valuable assistance in data and uncertainty analysis. We also thank P.J. Roache of Ecodynamics, Inc., Albuquerque, NM, and D.C. Wilcox of DCW Industries, Inc., La Canada, CA, for helpful discussions. This work was supported by the United States Department of Energy under Contract DE-AC04-94AL85000.

\section{References}

[1] "The Influence of Computational Fluid Dynamics on Experimental Aerospace Facilities: A Fifteen Year Projection," National Research Council, National Academy Press, Washington, D.C., 1983 .

[2] Bradley, R.G., "CFD Validation Philosophy," AGARD Symposium on Validation of Computational Fluid Dynamics, Paper No. 1, Lisbon, Portugal, 1988.

[3] Marvin, J.G., "Accuracy Requirements and Benchmark Experiments for CFD Validation," AGARD Symposium on Validation of Computational Fluid Dynamics, Paper No. 2, Lisbon, Portugal, 1988. 
[4] Martellucci, A., "The Challenging Process of Validating CFD Codes," AIAA Paper No. 90-1402, 16th AIAA Aerodynamic Ground Testing Conf., Seattle, WA, June 1990.

[5] Cosner, R.R., "Issues in Aerospace Application of CFD Analysis," AIAA Paper No. 94-0464, 32nd Aerospace Sciences Meeting, Reno, NV, Jan. 1994

[6] Oberkampf, W.L., "A Proposed Framework for Computational Fluid Dynamics Code Calibration/Validation," AIAA Paper No. 942540, 18th AIAA Aerospace Ground Testing Conf., Colorado Springs, CO, June 1994

[7] Neumann, R.D., "CFD Validation - The Interaction of Experimental Capabilities and Numerical Computations," AIAA 90-3030, 8th Applied Aerodynamics Conference, Portland, OR, Aug. 1990.

[8] Boehm, B.W., Software Engineering Economics, Prentice-Hall, 1981 .

[9] Blottner, F.G., "Accurate Navier-Stokes Results for the Hypersonic Flow over a Spherical Nosetip," J. Spacecraft \& Rockets, Vol 27, No. 2, pp. 113-122, Mar.-Apr. 1990.

[10] Mehta, U.B., "Flight Performance Estimation Utilizing Computational Fluid Dynamics," Proceedings of the 5th National Aerospace Plane Technology Symposium, Vol. 1, NASP CP-5028, Oct. 1988.

[11] Edwards, T.A., and Flores, J., "Computational Fluid Dynamics Nose-to-Tail Capability: Hypersonic Unsteady Navier-Stokes Code Validation," J. Spacecraft \& Rockets, Vol. 27, No. 2, Mar.-Apr. 1990, pp. 1213-130.

[12] Chapman, D. R., Mark, H., and Pirtle, M.W., "Computers vs. Wind Tunnels for Aerodynamic Flow Simulations," Aerospace America, Apr. 1975, pp. 22-35.

[13] Dwoyer, D., "The Relation between Computational Fluid Dynamics and Experiment," Invited Presentation, AIAA 17th Ground Testing Conference, Nashville TN, July 1992.

[14] Lynch, F.T., Crites, R.C., and Spaid, F.W., "The Crucial Role of Wall Interference, Support Interference, and Flow Field Measurements in the Development of Advanced Aircraft Configurations," $A G A R D C P$ 535, Wall Interference, Support Interference, and Flow Field Measurements, 73rd Fluid Dynamics Panel Symposium, Brussels Belgium, Oct. 1993, pp. 1-1-1-38.

[15] Ashill, P.R., "Boundary-Flow Measurement Methods for Wall Interference Assessment and Correction--Classification and Review," AGARD CP 535, Wall Interference, Support Interference, and Flow Field Measurements, 73rd Fluid Dynamics Panel Symposium, Brussels Belgium, Oct. 1993, pp. 12-1-12-21.

[16] Mason, W.H., "What We Need in Experimental Aerodynamics: One Engineering Educator's View," AIAA 92-0161, 30th Aerospace Sciences Meeting, Reno, NV, Jan. 1992

[17] Miles, R., Brown, G., Lempert, W., Natelson, D., Yetter, R., Guest, J., Williams, G., and Bogdonoff, S., "Radiatively Driven Hypersonic Wind Tunnel," AIAA 94-2472, 18th AIAA Aerospace Ground Testing Conf, Colorado Springs, CO, June 1994.
[18] Wilcox, D.C., Private Communication, 1995.

[19] Roache, P.J. "A Method for Uniform Reporting of Grid Refinement Studies," FED-Vol. 158, Quantification of Uncertainty in Computational Fluid Dynamics, ASME Fluids Engineering Conf., Washington, D.C., 1993, pp. 109-120.

[20] "Information for Contributors to Journals of the AIAA," American Institute of Aeronautics and Astronautics, 1995.

[21] Walker, M.M., and Oberkampf, W.L., "Joint Computational/ Experimental Aerodynamics Research on a Hypersonic Vehicle: Part 2, Computational Results," AIAA J., Vol. 30, No. 8, pp. 2010-2016, Aug. 1992.

[22] "Assessment of Wind Tunnel Data Uncertainty," AGARD Advisory Report, AGARD-AR-304, 1994.

[23] Coleman, H.W., and Steel, W.G., Experimentation and Uncertainty Analysis for Engineers, J. Wiley \& Sons, New York, NY, 1989.

[24] Coleman, H.W., Steele, W.G., and Taylor, R.P., "Implications of Correlated Bias Uncertainties in Single and Comparative Tests," ASME $J$. Fluids Engineering, to be published.

[25] Brown, K.K., Coleman, H.W., Steele, W.G., and Taylor, R.P., "Evaluation of Correlated Bias Approximations in Experimental Uncertainty Analysis," AIAA Joumal, to be published.

[26] "Aerodynamic Data Accuracy and Quality: Requirements and Capabilities in Wind Tunnel Testing," AGARD CP-429, Sept. 1987.

[27] Oberkampf, W.L., and Aeschliman, D.P., "Joint Computational/ Experimental Aerodynamics Research on a Hypersonic Vehicle: Part 1, Experimental Results," $A I A A$ J., Vol. 30 , No. 8, pp. 2000-2009, Aug. 1992.

[28] Aeschliman, D.P., Oberkampf, W.L., and Henfling, J.F., "FastResponse, Electronically-Scanned Multi-Port Pressure System for Low-Pressure Hypersonic Wind Tunnel Applications," AIAA Paper No. 94-2580, 18th AIAA Aerospace Ground Testing Conf., Colorado Springs, CO, June 1994.

[29] Reda, D.C., and Aeschliman, D.P., "Liquid Crystal Coatings for Surface Shear Stress Visualization in Hypersonic Flows," J. Spacecraft \& Rockets, Vol. 29, No. 2, Mar-Apr. 1992, pp. 155 158.

[30] Steger, J.L., Ying, S.X., and Schiff, L.B., "A Partially FluxSplit Algorithm for Numerical Simulation of Compressible Inviscid and Viscous Flow," Proceedings of the Workshop on Computational Fluid Dynamics, Inst. of Nonlinear Sciences, Univ. of California, Davis, CA, 1986.

[31] "INCA: 3D Multi-Zone Navier-Stokes Flow Analysis with Finite-Rate Chemistry, User's Manual Part 1: Input Guide," Amtec Engineering, Inc.; Bellevue, WA, Jan. 1992."

[32] Payne, J.L., and Walker, M.A., "Verification of Computational Aerodynamic Predictions for Complex Hypersonic Vehicles using the INCATM Code," AIAA 95-0762, 33rd Aerospace Sciences Meeting, Reno, NV, Jan. 1995. 
[33] Noack, R.W., and Lopez, A.R., "Inviscid Flow Field Analysis of Complex Reentry Vehicles: Vol. 1, Description of Numerical Methods," Sandia National Labomtories Report SAND87-0776,

Oct. 1988.

Table 1: Summary of Results for Uncertainty Analysis

\begin{tabular}{|c|c|c|c|c|c|c|c|c|}
\hline \multirow{2}{*}{$\begin{array}{c}\text { Uncertainty } \\
\text { Type }\end{array}$} & \multicolumn{2}{|c|}{$\mathrm{C}_{\mathrm{n}}$} & \multicolumn{2}{|l|}{$\mathrm{C}_{\mathrm{m}}$} & \multicolumn{2}{|c|}{$x_{c p^{\prime}} L$} & \multicolumn{2}{|c|}{$\mathrm{C}_{\mathrm{a}}$} \\
\hline & $\widehat{\sigma}$ & $\%$ & $\hat{\sigma}$ & $\%$ & $\widehat{\sigma}$ & $\%$ & $\hat{\sigma}$ & $\%$ \\
\hline Instrument & $0.474 \times 10^{-3}$ & 20 & $0.406 \times 10^{-3}$ & 19 & $0,413 \times 10^{-3}$ & 9 & $0.426 \times 10^{-3}$ & 63 \\
\hline Flow* & $0.941 \times 10^{-3}$ & 80 & $0.851 \times 10^{-3}$ & 81 & $1.322 \times 10^{-3}$ & 91 & $0.324 \times 10^{-3}$ & 37 \\
\hline Total & $1.054 \times 10^{-3}$ & 100 & $0.943 \times 10^{-3}$ & 100 & $1.385 \times 10^{-3}$ & 100 & $0.535 \times 10^{-3}$ & 100 \\
\hline
\end{tabular}

* Axial flow gradient and mirror symmetry flow components
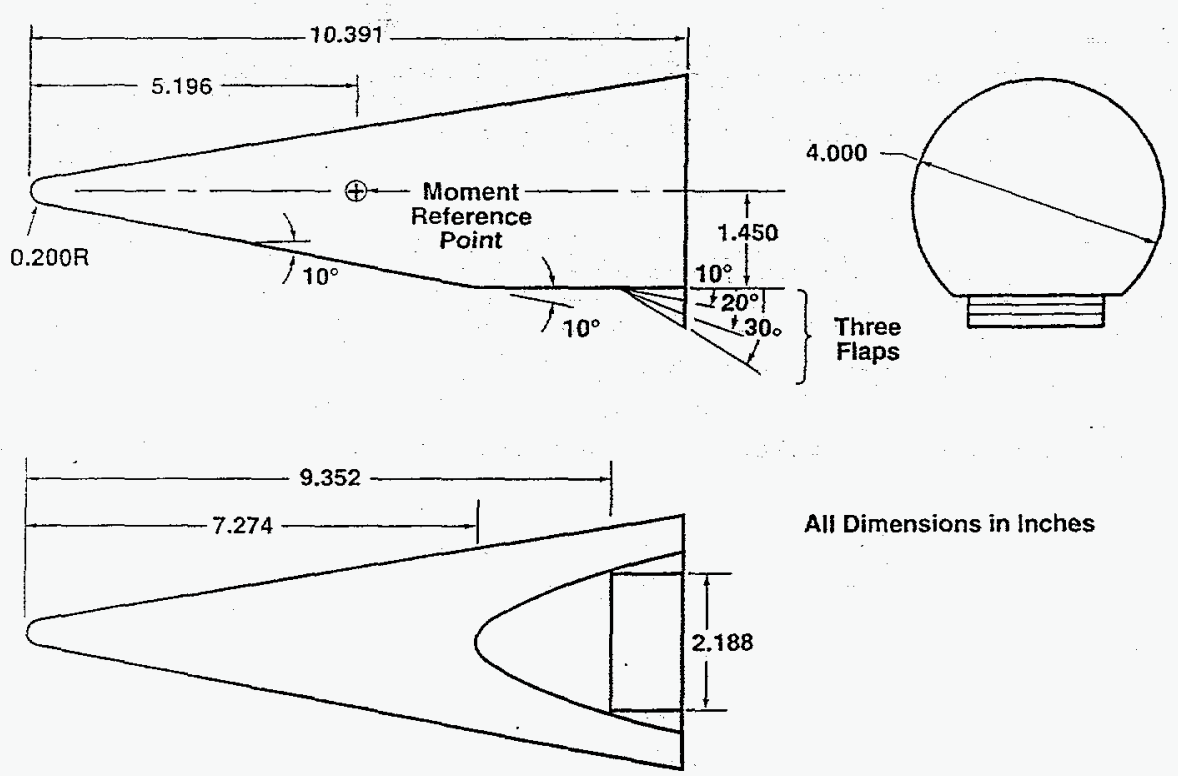

All Dimensions in lnches

Fig. 1. Variable-geometry sliced sphere/cone hypersonic wind tunnel model for CFD code validation research. 


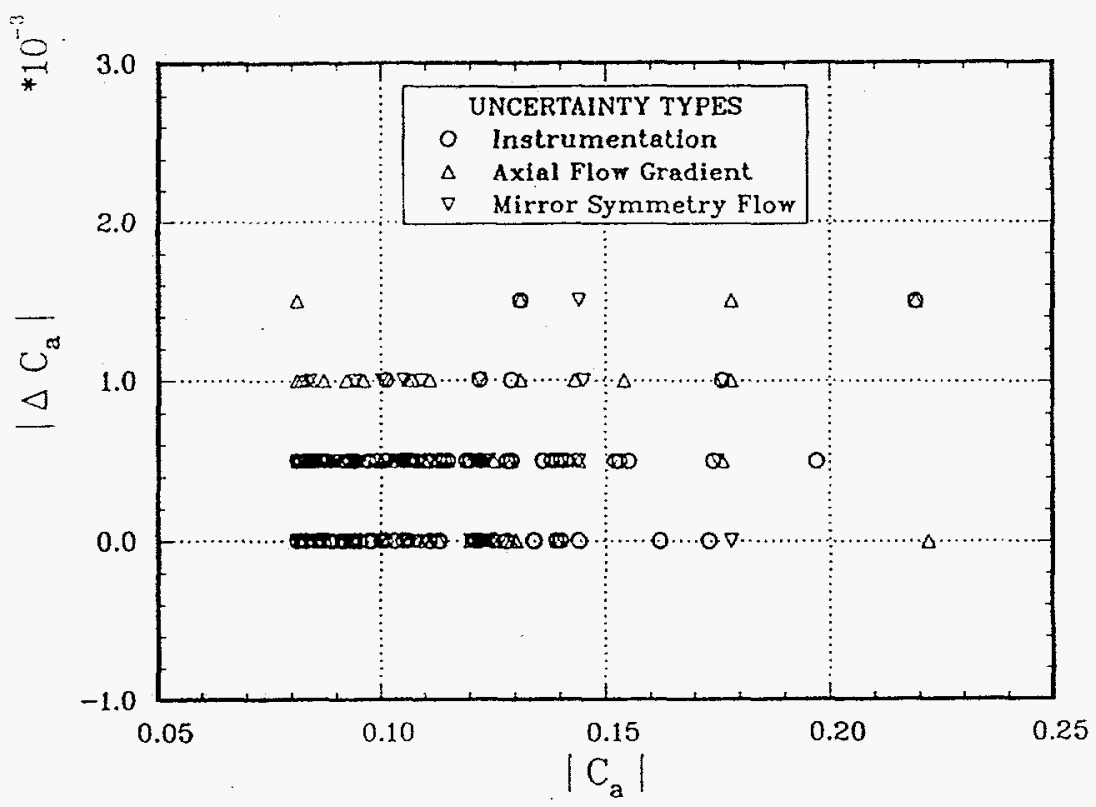

Fig. 2. Residuals, $\Delta C_{a}$, of experimental measurements for axial force coefficient.

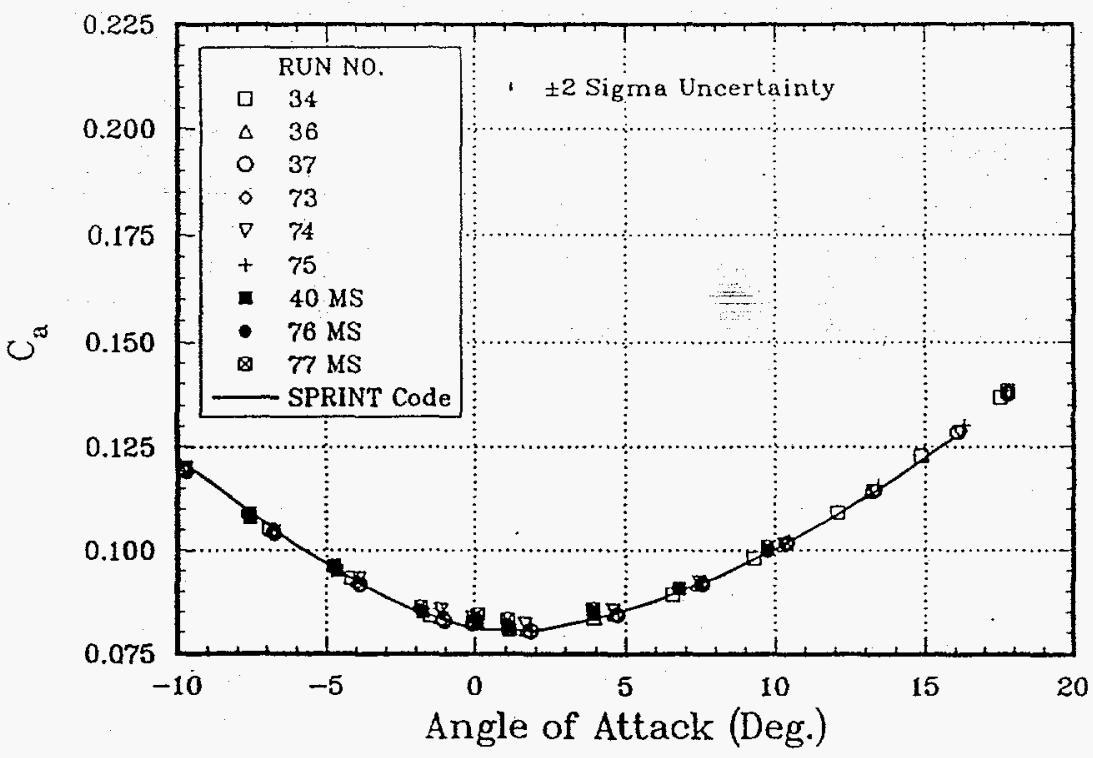

Fig.3. Experimental and computed axial force coefficient, $\mathrm{C}_{\mathrm{a}}$, for slice-only model configuration vs angle of attack for zero roll angle. 


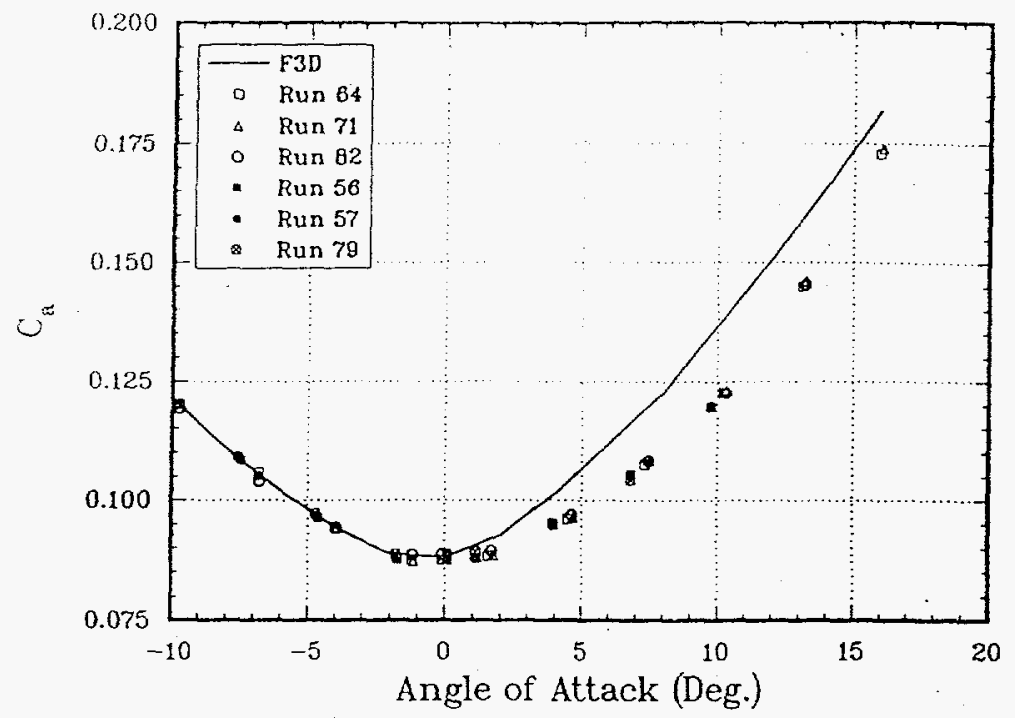

Fig. 4. Experimental and computed axial force coefficient; $\mathrm{C}_{\mathrm{a}}$, for $30 \mathrm{-deg}$ flap model configuration vs angle of attack for zero roll angle.

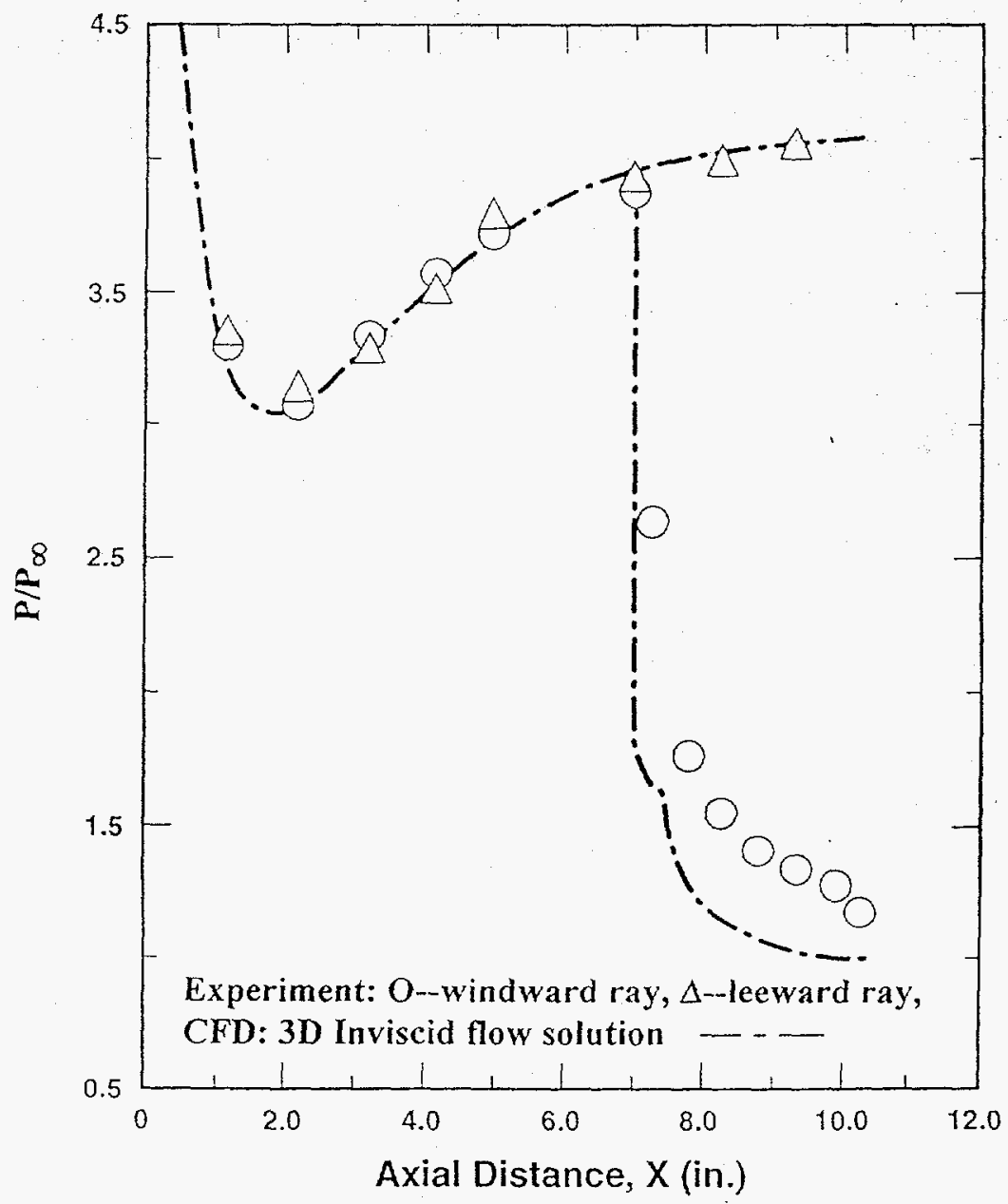

Fig. 5. Experimental and computed surface pressure coefficient, $\mathrm{P} / \mathrm{P}_{\infty}$, along windward and leeward rays for sphere/cone model in sliceonly configuration at zero angle of attack. 